\title{
ScIDoC
}

International Journal of Behavioral Research \& Psychology (IJBRP)

ISSN 2332-3000

\section{Suicide a Menace in Secondary Schools: A Case of Gusii Region, Kenya}

Review Article

Nyamwange $C^{*}$

Senior Lecturer, Educational Psychology, Kisii University, Kenya.

Director, Calsams \& Gidkemtons Consultancy Firm.

Director, Bright Future Wellness Center, Milimani, Kisii.

\section{Abstract}

More than 800,000 people die by suicide every year around one person every 40 seconds. Suicide occurs all over the world and can take place at almost any age. Globally, suicide rates are highest in people aged 70 years and over. In some countries, however, the highest rates are found among the young. Worryingly, suicide is the second leading cause of death in 15-29 year olds globally. Suicide has become a menace in Kenya with rates going high by the day. This study was carried out among the Gusii people of Kenya which targeted secondary school students. The purpose of this study is to establish if students of secondary school have social interactions, friends, peers, relationships and feel burdensome to parents by secondary school students as this are predictors of suicide. The study utilized a qualitative research design and data was collected by use of questionnaires and data was analyzed by use of simple percentages The study revealed that majority of the students $70 \%$ of the respondents said that they spend time with friends at home and at school and $21 \%$ do not enjoy spending time with family or even with friends whereas $9 \%$ remained neutral. On how they feel with their peers $40(26 \%)$ feel comfortable majority $99(66 \%)$ do not like the idea of being with peers. The study gave recommendations based on the findings of what needs to be done to reduce the predictors of suicide.

Keywords: Suicide; Student; Peer; Relationship; Family; Friendship.

\section{Background}

Suicide is a complex phenomenon that emerges out of a dynamic interaction involving biological, psychological, social, cultural, and spiritual factors. Suicide is most often the result of profound pain, hopelessness and despair: it is the triumph of pain, fear and loss over hope (Bertolote, 2000) [1].

Suicide is one of the top ten leading causes of death in the United States and was responsible for almost 45,000 deaths in 2016. Many more people are hospitalized as a result of nonfatal suicidal behavior (i.e., suicide attempts) than are fatally injured, and an even greater number are either treated in ambulatory settings (e.g., emergency departments) or not treated at all [1, 2]. Among adults aged 18 years and older, for each suicide there are about 30 adults who reported making a suicide attempt. In the United States, suicide was responsible for 44,193 deaths in 2015, which is approximately one suicide every 12 minutes (CDC, 2015) [2].

Suicide, like other human behaviors, has no single determining cause. Instead, suicide occurs in response to multiple biological, psychological, interpersonal, environmental and societal influences that interact with one another, often over time. According to CDC (2015) [2] the social ecological model-encompassing multiple levels of focus from the individual, relationship, community, and societal-is a useful framework for viewing and understanding suicide risk and protective factors identified in the literature.

WHO (2018) [11] Suicides happen in all countries and regions, whether rich or poor. However, most occur in low and middleincome countries, which accounted almost four-fifths of global suicides in 2016. Every year, close to 800000 people die due to suicide every year, it is estimated that the method used for 20 percent of global suicides is pesticide self-poisoning, most of which occur in rural agricultural areas in low- and middle-income

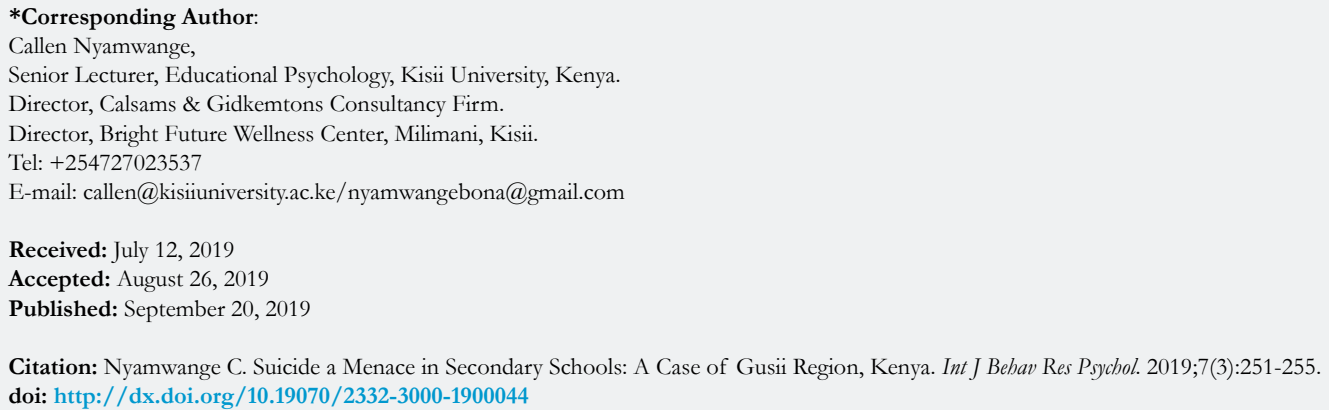

Copyright: Nyamwange $\mathbf{C}^{\circ}$ 2019. This is an open-access article distributed under the terms of the Creative Commons Attribution License, which permits unrestricted use, distribution and reproduction in any medium, provided the original author and source are credited. 
countries. Other common methods of suicide are hanging and firearms. In high-income countries, there is a well-established link between suicide and mental health issues such as depression and alcohol use disorders, but many suicides take place on an impulse, during moments of crisis.

Standard digital (2019) [8] Central Kenya region is losing at least five lives every day through suicide in what officials and residents say is a never seen-before catastrophe that should now trigger community reawakening to restore social ethics.

The worst hit, according to police records availed to Mt. Kenya Star is Nyeri County where on average, four people are killing themselves every day.

Kirinyaga follows with at least one death per day while in Murang'a at least four people commit suicide every week.

Despite the grave consequences of the matter, political leaders and especially the governors are yet to mainstream the issue on the region's debate, leaving the burden to the regional administration and the church.

WHO (2019) [9] Suicide cases have been on the rise here in Kenya, according to WHO the number of suicides reported in Kenya rose by 58 per cent between 2008 and 2017 to reach 421 . The report also shows that more men are likely to commit suicide than women. Out of the 421 suicide cases in 2017, 330 involved men. Within Universities here in Kenya, there have been a worrying statistic of students committing suicide. Universities have been reluctant in sharing statistics however there have been slight over twenty documented cases of students who have committed suicide.

TUKO (2019) [3] Suicide cases have been on a steady increase raising concern across the country - Police records show that at least 80 people took their own lives in the past one month - This was attributed to stress, depression and mental illnesses - Some of those who took their own lives were mourning deaths of their spouses At least 80 people have committed suicide in the country in the last four weeks according to police records. Five of the victims were facing serious criminal cases including murder, attempted murder and stealing and all committed suicide while in police custody.

Kegochi the district commissioner, Kisii counties on suicide in Kisii noted "The cases of men taking their own lives in Kisii are worrying. My appeal to men is that they should guidance and counseling anytime they are faced with difficulties in life," Kegochi stated. Junior (2019) The commissioner challenged local chiefs to conduct weekly baraza in their respective places of jurisdiction to advise the public accordingly. He noted that losing men prematurely is increasing the number of orphans and widows in the region.

MHA (2018) [5] A suicide attempt is a clear indication that something is gravely wrong in a person's life. No matter the race or age of the person; how rich or poor they are, it is true that most people who die by suicide have a mental or emotional disorder. The most common underlying disorder is depression, $30 \%$ to $70 \%$ of suicide victims suffer from major depression or bipolar (manicdepressive) disorder. It is estimated that around $20 \%$ of global suicides are due to pesticide self-poisoning, most of which occur in rural agricultural areas in low- and middle-income countries. Other common methods of suicide are hanging and firearms. Knowledge of the most commonly used suicide methods is important to devise prevention strategies which have shown to be effective, such as restriction of access to means of suicide.

Gregory.C. (2019) [3] notes that The most cited risk factors for suicide include psychiatric disorders, genetics, co-exist. Access to weapons and other methods of suicide also increase risk. For example, rates of suicide in homes with guns are greater than in homes without them. Mental disorders play an overwhelming role in the increased risk of suicide - with estimates suggesting up to $90 \%$ of individuals who take their own life suffer from some type of psychiatric disorder. Risk of suicide for individuals suffering from mental disorders drastically decreases once admitted to treatment The mental disorders with the greatest prevalence of suicide risk associated with them include major depressive disorder, bipolar disorder, schizophrenia, personality disorders, post traumatic stress disorder, and eating disorders. Individuals suffering from major depressive disorder and bipolar disorder are at the highest risk of suicide - with risk of suicide increasing 20-fold. Gregory.C. (2019) [3].

Behind major depressive disorder and bipolar disorder, substance abuse ranks as the second highest risk factor for suicide. Statistics indicate that alcoholism is present at the time of death in up to $61 \%$ of completed suicide cases. Heroin and cocaine use is also a common risk factor for suicide, with heroin users having a 14fold greater risk of suicide and cocaine users having a higher risk of suicide during withdrawal drug use. Cannabis use has not been found to increase suicide risk among users. Gregory.C. (2019) [3].

Genetics is thought to play a role in risk of suicide - such that a family history of suicide tends to indicate an increased risk of suicide among other family members - accounting for up to $55 \%$ of suicidal behaviors. Family history of mental disorders and substance abuse is also a risk factor for suicide. In a similar respect, exposure to suicide (e.g., watching a family member commit suicide or finding their body) is also indicative of an increased risk of suicidal behavior.

Family and socio-economic problems are also contributing factors to suicide risk. Unemployment, homelessness, poverty, childhood sexual abuse, social isolation, loss of a loved one, and other life stresses can all increase the likelihood of suicide. Sexual abuse alone is thought to contribute to $20 \%$ of the overall risk of suicide.

\section{Rates Suicide Rates by Age Gregory.C. (2019)}

In 2017, the highest suicide rate (20.2) was among adults between 45 and 54 years of age. The second highest rate (20.1) occurred in those 85 years or older. Younger groups have had consistently lower suicide rates than middle-aged and older adults. In 2017, adolescents and young adults aged 15 to 24 had a suicide rate of 14.46. Gregory.C. (2019) [3].

\section{Suicide Rates by Race/Ethnicity}

In 2017, the highest U.S. age-adjusted suicide rate was among Whites (15.85) and the second highest rate was among Ameri- 
can Indians and Alaska Natives (13.42). Much lower and roughly similar rates were found among Black or African Americans (6.61) and Asians and Pacific Islanders (6.59).

Note that the Center for Disease Control and Prevention (CDC) records Hispanic origin separately from the primary racial or ethnic groups of White, Black, American Indian or Alaskan Native, and Asian or Pacific Islander, since individuals in all groups may also be Hispanic.

WHO (2012) [10] A comprehensive approach to suicide prevention and intervention - characterized by multiple strategies implemented across an array of key contexts, over time - is likely to yield the greatest benefit in reducing the incidence of suicide and suicidal behaviour. The delivery of high quality assessment and treatment services, focused on both reducing the risk factors for suicidal behavior, while simultaneously bolstering protective factors, is a central component in overall suicide prevention efforts.

(CDC, 2015) [2] Suicide and nonfatal self-directed violence result in an estimated $\$ 69$ billion in combined medical and work loss costs [1]. However, because that estimate does not include other societal impacts (like those on families), the true cost of suicide is likely much higher.

\section{Gender Differences}

NCH (2019) [6] Suicide is the second leading cause of death among youth aged 10-19 years in the U.S., with rates historically higher in males than females. However, recent reports from the Centers for Disease Control and Prevention reveal female youth are experiencing a greater percent increase in suicide rates compared to males. Bridge said females have higher rates of non-fatal suicidal behavior, such as thinking about and attempting suicide, but more males die by suicide than females. "One of the potential contributors to this gender paradox is that males tend to use more violent means, such as guns or hanging," said Bridge. "That makes the narrowing of the gender gap in suicide by hanging or suffocation that we found especially concerning from a public health perspective."

\section{Prevention}

Suicide prevention methods and treatment are based on patient risk factors. Treatments are prescribed in light of underlying conditions in addition to prevention of suicidal thoughts and acts. If you are suffering from a mental disorder, a treatment plan to treat this condition is implemented first. One of the most common suicide prevention techniques is psychotherapy - also known as talk therapy - in the form of Cognitive Behavioral Therapy (CBT) or Dialectical Behavior Therapy (DBT) (Gregory, 2019) [3].

MHA (2018) [5] Cognitive Behavioral Therapy is a common treatment option for individuals suffering from a variety of mental disorders. In this method of psychotherapy, you are taught new ways of dealing with stress and stressful life experiences. In this manner, when thoughts of suicide arise, you can redirect those thoughts and cope with them in a different way than attempting to take your own life.

Dialectical Behavior Therapy is used to help an individual recognize disruptive or unhealthy feelings or actions. In relation, this therapy method then introduces techniques on how to deal with difficult or troubling situations. More research is needed on psychotherapy related to suicide prevention though, as DBT, in particular, has been shown to decrease the prevalence of attempted suicide but has shown no effect on completed suicides.

Medications can also be prescribed as a prevention method to suicide; however, controversy exists in this method, as many medications used in the treatment of mental disorders include increased risk of suicide as a side effect. Antidepressants especially carry a risk of potential increase in suicidal thoughts and behavior but this risk might be dependent on age. Clinical research has shown that young adults increase their risk of suicide and suicidal thoughts when taking antidepressants, but in older individuals, this side effect diminishes.

Sheikhmoonesi and Zarghami ( 2014) [7] Increased awareness among doctors is also a prevention technique. Research indicates that many individuals who have completed suicide or attempted suicide did seek medical attention in the year prior; however, warning signs may have been missed. Increased education and awareness among medical professionals might decrease suicide rates in the future.

WHO (2018) [9] Popular "crisis hotlines" have not received solid data indications in the research that suggest their use is effective or not. Though, one positive side effect of these hotlines is that they are generally well-known and common - increasing the general population's awareness of suicide. In an additional effort to bring awareness to suicide and risk factors associated with suicide, September 10 has been observed as World Suicide Prevention Day in partnership with the International Association for Suicide Prevention and the World Health Organization.

\section{Methodology}

The Republic of Kenya is a country in Eastern Africa, with a population of approximately 41.61 million people. It lies on the equator and is bordered by Ethiopia (North), Somalia (East), Tanzania (South), Uganda (West), and Sudan (Northwest), with the Indian Ocean running along the southeast border. It has an extension of 582,646 sq. $\mathrm{km}$. The country is named after Mount Kenya - before 1920, the area now known as Kenya was known as the British East Africa Protectorate. Kenya is a diverse nation of 42 distinct ethnic groups. Official languages are Swahili and English and the currency is Kenyan Shilling. The exact place the study was carried out among the larger Gusii people that are surrounded by The Luo to the southand Kipsigi to the North Masaais to the Eastern. The paper used primary data sources to investigate the state of suicidal ideation among secondary school students both males and females. The sample size consisted of only 30 students of Form three from each of the five (5) schools selected. These respondents were sampled by use of simple random sampling. The study adopted qualitative approach design of analysis of data.

\section{Findings}

The study sought to find out why cases of suicide were on the rise in Kenya with reference to Kisii county. The respondents were asked several questions ranging from pre-disposing factors, signs and symptoms and possible interventions. 
Social interaction is an exchange between two or more individuals and is a building block of society. By interacting with one another, people design rules, institutions and systems within which they seek to live. The respondents were asked information on social interactions in school and home. Majority $70 \%$ of the respondents said that they spend time with friends at home and at school, they hate those who use drugs and abusive language, engage in church activities, Positive with life and Focuses in academic matters, enjoy good family relations, spend time composing songs, are disciplined and comfortable at home and school, ready to interact with all manner of people regardless of age, gender and social class and enjoy learning more from friends and copies the right things only has a slogan "copy character admire only". A small number $21 \%$ said that they have no interactions with anyone whether in the school or at home instead interacts with electronics, Keep to themselves both at school and at home, Solve their problems alone and occasionally with the mothers, Difficult in interacting with others, Have cordial relationships with people though shy sometimes, interactes with various people some who feel worthless, Not happy with interactions at home as they are accuse falsely, Like socializing with the opposite gender and those older than them, silent and avoid trouble (withdrawn), do not like interacting as they feel uncomfortable and see everybody as a nobody to them. An average of $9 \%$ did not respond to the question.

On the kind of friends they keep, the respondents 69\% said they have Self driven friends, have as few as two friends, they are outgoing friends and like visiting urban areas when idle, God fearing friends caring and hard working and had best friends while in primary but do not have in high school. $19 \%$ of respondents said many of their activities centered around home, have less than 10 friends, engage in church activities with many friends but doesn't maintain intimacy and like watching movies and listening to music. Students who have friends they confide in are likely to sort out issues before hand unlike those who do not keep friends and $12 \%$ did not respond to the question.

Friends are important. So important, in fact, that it's been proven that friendship can extend life expectancy and lower chances of heart disease. Friendship helps us survive. ... Friendship ignites the part of the brain that makes us feel good, which makes us want to keep hanging out with our friends and by doing so one is able to speak out the bad feeling that can lead to suicide.

On how they feel with their peers $40(26 \%)$ respondents said that they feel comfortable with peers, find hope with peers, motivated because of peers, feels great with peers, find security with the group, learn a lot from the peer group, feel confident and loved, feels equal and they feel proud because they are one. Majority $99(66 \%)$ of learners do not like the idea of being with peers as they reported feeling lonely sometimes, feel unhappy sometimes because they do not belong to any group instead maintain one or no friend, do not belong to any peer group, feel disappointed in the company of peer group because they gossip a lot about immorality, engage in theft, sometimes feel left out especially when not updating on trending issues, feel that they are not fit in the company of peers because they engage in different things that do not interest them, feels they make her have low self esteem by embarrassing them and abusing them, They speak/utters words which lowers their self esteem as they ridicule when being corrected, feel discouraged because some of them abuse alcohol and drugs, feel isolated because they come from rich families and get irritated with their behavior and many of them don't respect others or even teachers. 11(7.2\%) respondents did not respond to the question which accounts for $16 \%$ of the total population. Students who find difficult to being with the peers are potential victims of suicide if not checked.

When comparing responses based on gender the study revealed that boys are more vulnerable to suicidal feeling as their responses were sometimes many felt misled by peers who do against expectations, feels like the peer can lead them to commit suicide by negative influences, finding it difficult to resist from their negative influence, sometimes feel despised when they do things they don't like doing. Many feel isolated from peers making them avoid the group which is not common among girls.

On relationship with family members a majority of respondents $107(71.3 \%)$ said they engage in Unhelpful family relationship hence feel bitter about it, do not interact with family members, non-cooperative family making them regret why they live, non united family, an uncaring fathers, parents are too harsh, parents keep on disagreeing and they fight, Conflicts between parents because of financial issues, close relationship with mother but not with father and Close with mother and siblings but not close with father. These students feel challenged because the fathers are not concerned about the school fees and generally the basic necessities only the mothers who is struggle with them. They find themselves lonely because the fathers are deceased and one reported being the being the only male in the family he finds it hard to share with siblings as the father rebukes him and they noted that have family members that are Jealousy don't wish good for them. Those respondents who enjoy positive family relationship 34 $(22.6 \%)$ said, they enjoy free and supportive family, close loving family, free interaction with family, Helpful, supportive, understanding, peaceful, enjoy a strong bond, basic needs are met and family members are caring. A total of 9 did not respond to the question accounting for $9(6 \%)$.

Students who experience family cohesion and expressiveness are stronger and may not develop suicide ideation than family where conflict is a day today affair. Unreachable parents are the kind of parent-child relationship that can lead to suicidal risk because the parents do not play their role as a pattern to imitate by their off spring. It happens that the extraordinary achievements expected by parents are not achievable and the parents keep off. By keeping off their children negative feelings of unsuitableness, poor self-esteem and self-sufficiency for not having been as successful which in most cases develop to suicidal ideation.

When asked if they felt being a burden to family and to friends, majority $90(60 \%)$ responded thus; yes by going against family expectations and engaged in immorality, parents are not able to provide basic needs, poor performance, parents have never appreciated their grades, parents say that they are too demanding, lacks love from friends, parents lack of money so cant satisfy their needs, discrimination among siblings by parents, discrimination by friends who feel they do not have anything to offer, lack of money due to the poverty parents experience hence struggle to give basic needs, lack of trust appreciation, do not behave well, fails in exams yet parents struggle to pay fees and for some parents are separated and their situation is deplorable and abandoned. 60 (40\%) respondents gave no as an answer meaning they do not feel 
being a burden to their parents or friends.

Students who feel being a burden to family or even to friends are likely to develop negative feeling and sometimes suicidal as a means of escape from such thoughts. Such students need to be supported so as to get out of suffering otherwise suicide can be the next option as suicide is a desperate attempt to escape suffering that has become unbearable. Blinded by feelings of selfloathing, hopelessness, and isolation, a suicidal person can't see any way of finding relief except through death.

\section{Conclusions}

From the foregoing information on suicide it has come out clearly that social interaction help in preventing suicidal ideation and family and friendship is an important aspect in supporting positive growth to the students in schools. Students must be engaged so that they have no free time to engage in thoughts that are negative. Parents and schools to collaborate in meeting the basic needs of survival for students at home and at school.

\section{Recommendations}

- Schools have a responsibility of helping those students who like keeping to themselves as they easily become victims of suicidal ideations.

- Parents need to take responsibility of providing basic needs of their children especially paying their fees, provision of shopping, pocket money and appreciate them even when they have not performed well in school as expected.

- Students to be encouraged to participate in peer group activities as interaction is a health thing for any human being.

- Guidance and Counseling have a significant role in the assessment, clinical management, and treatment of students who are at risk for suicide.

\section{References}

[1]. Bertolote J.M. World Health Organization. Preventing suicide: a resource for primary health care workers. Geneva: World Health Organization; 2000.

[2]. CDE. Suicide Policy Brief: Preventing Suicide In Rural America, USA, CDC Stacks; 2015.

[3]. GregoryC. Suicide and Suicide Prevention: Understanding the risk factors, prevention, and what we can do to help, USA; 2019.

[4]. Lynda Monk. L MSW, RSW Samra. J, Rpsych. Working With the Client Who is Suicidal: A Tool for Adult Mental Health and Addiction Services; 2018.

[5]. MHA Suicide, No suicide attempt should be dismissed or treated lightly! Why Do People Attempt Suicide? USA; 2018.

[6]. NCH. Study finds narrowing gender gap in youth suicides, JAMA Network Open; 2019.

[7]. Sheikhmoonesi. F, Zarghami. M. Prevention of Physicians' Suicide: Iran J Psychiatry Behav Sci. 2014 Summer; 2014: 8(2): 1-3.

[8]. Standard digital. Five suicide deaths reported in Central Kenya every day, Mt Kenya Star ; 2019.

[9]. WHO. UN NEWS: Global Perspective; Human Stories; 2018

[10]. WHO. public health action for the prevention of suicide, WHO Library Cataloguing-in-2019; 2012.

[11]. World Health Organization. National suicide prevention strategies: Progress, examples and indicators, ISBN 978-92-4-151501-6; 2018. 